Lepr Rev (1992) 63, Supplement, 114s-122s

\title{
Report on the group discussions on the needs and prospects for epidemiological tools in leprosy control
}

\section{State of the art on the epidemiology of leprosy}

\subsection{INTRODUCTION}

The major gaps in the knowledge on the epidemiology of leprosy in relation to control and, consequently, recommendations for epidemiological research and for health systems research were identified.

The recommendations for epidemiological research in leprosy have been divided into the epidemiology of infection, disease and disability. New studies will be required to address some of the recommendations, but, wherever possible, existing data sets (including vaccine trials, drug trials and good control programmes) should be exploited.

\subsection{EPIDEMIOLOGY OF INFECTION}

There is a continued need for a sensitive and specific assay for Mycobacterium leprae infection. New developments in PCR technology and in immunodiagnostic tests may ultimately permit studies of the distribution and determinants of infection in communities, thereby identifying potential sources of transmission, and groups at particular risk of disease, and allowing transmission to be monitored. Eff orts should be made to encourage relevant basic research towards such tools, and to facilitate their rapid evaluation in field contexts.

\subsection{EPIDEMIOLOGY OF DISEASE}

1.3.1 The known determinants of clinical leprosy include age, sex, household contact, geographical location, genetics, BCG and 'socioeconomic factors'. It may be useful to express the associated risks in terms of attributable risks, to define the proportion of clinical cases associated with various factors, for example, what proportion of cases in various communities are attributable to household contact.

1.3.2 The relative importance of intra- versus extra-household contact as a risk factor may change (increase?) as leprosy declines. This could have practical implications for the role of (household) contact surveys in leprosy control. The extent to which present and past household contact with cases, and different family relationships to cases, affect the risk of leprosy thus should be studied in different epidemiological contexts. Efforts should be made to define the proportion of cases which might be 
identified by expanding the definition of contact to include, for example, past household contact and distant relations to known cases.

1.3.3 It is important to analyse the distribution of cases by place of birth as well as by place of residence at onset of disease or registration. These distributions should be interpreted with relation to a control group and/or to information on patterns of migration within the population concerned. This is particularly important for urban areas.

1.3.4 The implications of HIV infection for leprosy (in particular multibacillary disease) incidence, reaction and relapse should be further evaluated. Case control studies are appropriate, with attention to confounding, for example by nonleprosy morbidity and by social factors.

1.3.5 Socioeconomic factors and different ecological conditions are obviously important in leprosy. Efforts should be made to distinguish between various factors associated with low socioeconomic status, for example overcrowding, which may be associated with leprosy risk.

1.3.6 Efforts should be made to identify (immunological) correlates of BCG-induced protection, in order to explain observed differences in vaccine efficacy. The identification of such correlates would have great utility both in predicting the efficacy of BCG in different populations and for the screening of potential vaccine reagents.

1.3.7 The cost-effectiveness of different vaccines and different vaccination strategies, different treatment regimens and treatment strategies including new drugs should be investigated. The use of epidemiological models should form part of the evaluation of cost-effectiveness.

\subsection{EPIDEMIOLOGY OF DISABILITIES}

Leprosy associated disability is a critically important but understudied aspect of leprosy epidemiology and control. Descriptive studies of the patterns of disabilities in representative samples or populations of leprosy patients should be encouraged. Even more useful would be longitudinal studies of the natural history of, and risk factors for, reactions and their outcome, and clinical trials of various interventions to control reaction and to prevent or reverse disabilities.

\subsection{HEALTH SYSTEMS RESEARCH}

Leprosy programmes at different levels face operational problems in implementing control. Problem-solving has often been based on nonsystematic and intuitive approaches. It is here that health systems research (HSR) could play an important role by introducing scientific methods for acquiring information which can be used for rational decision making at the operational level. HSR is multidisciplinary, which means that programme managers responsible for such research should seek collaboration from appropriate experts such as social scientists before embarking on HSR. HSR is participatory so that persons concerned and dealing with the problem participate in the research projects. HSR should lead to action and results must be utilized for decision making. 


\section{Prediction of future trends in leprosy}

\subsection{NEEDS FOR THE PREDICTION OF FUTURE TRENDS}

The ability to predict future epidemiological trends is an important requirement for disease control programmes. At present this is particularly important for leprosy control because of the rapidly changing epidemiological situation as a result of the introduction of MDT. The prediction of trends in leprosy is required for the following purposes.

\subsubsection{Planning}

The prediction of trends in the prevalence of registered cases and in the incidence of new leprosy cases provides a time-frame for future epidemiological changes and a basis for the planning of resources. The estimation of the benefits of control and resource requirements will help to mobilize sustained political and funding commitments in the long term.

\subsubsection{Evaluation}

The comparison of predicted and observed trends facilitates the interpretation of evaluation results in terms of effectiveness of control, both at the global and the local level. The cause of possible unsatisfactory results can be analysed and the control strategy adjusted accordingly.

\subsubsection{Research}

Prediction techniques such as modelling can be a powerful tool to improve the comprehensive understanding of the quantitative aspects of transmission, disease and the impact of control. Another application is the prospective evaluation of alternative control strategies to identify the optimal approach. Prediction of the impact of potential new tools for control, such as vaccination and immunodiagnosis, may help to secure the required funding for the development of such tools.

\subsection{METHODS FOR THE PREDICTION OF FUTURE TRENDS}

A series of methods are applicable.

\subsubsection{Extrapolation}

Conventional extrapolation has until now been the standard method for prediction. Extrapolation should preferably be based on incidence rates (or case-detection rates, adjusted to the extent possible for the delay between onset and registration). Extrapolation of prevalence rates will often be misleading.

\subsubsection{Proxy trend indicators}

In many areas, population based rates are unavailable or cover only a very short period of time. In such situations, the need arises for a quick assessment of secular trends based on 
rather rough data. Previous analyses have shown that age at onset, the proportion of multibacillary cases and sex ratio can be used as proxy indicators of changing trends.

\subsubsection{Simulation modelling}

Simulation modelling combines demography, epidemiology and control measures in one coherent framework. This makes these methods appropriate for the assessment and adjustment of control measures. This includes the explanation of deviations from predicted trends as a result of differences between executed and planned control policies. Computer simulation models can also be useful in optimization by studying the combined impact of different control methods (vaccination, MDT, early case-detection, rehabilitation) on population health.

\subsubsection{Expert opinion (Delphi)}

Expert opinion, such as the Delphi technique, is necessary when data are lacking. These methods are being used increasingly in health planning but they are of limited value for quantitative predictions of trends.

\subsection{REQUIRED DATA FOR TREND PREDICTIONS}

To enable the prediction of trends, the following data are required: Minimal requirement (data to be collected at registration):

-Date of registration

-Date of birth (or estimated year of birth)

-Sex

-Place of residence

-Multibacillary or paucibacillary

If feasible, the following data should also be collected:

- Stated year of onset

-Disability at registration

-Date of start of treatment

- Type of treatment

- Treatment compliance

-Date of discharge or death

-Reasons for discharge

-Date of relapse

- Treatment of relapse

-Date of discharge after relapse

Information on the progression of disability would be of additional value for estimating the public health importance of leprosy.

Preferably all methods should be based on incidence rates necessitating population data on age and sex. However, in the lack of such data, proxy indicators could be based on proportions calculated on the basis of the patients registered. 


\subsection{CONCLUSION}

A situation of declining incidence rates together with the large scale introduction of MDT calls for the utilization of methods for the prediction of future trends. Conventional extrapolation is the simplest prediction method but it is limited in scope. Methods based on proxy indicators give more refined predictions and better possibilities for interpretation. Simulation modelling, the most complex method, can in addition address questions about changing epidemiological trends resulting from the effect of control measures applied to the target population.

Given the importance of reliable trend predictions, the considerable expenditures involved in collecting large amounts of evaluation data, and the relatively modest investments required for further development and application of prediction methods, the meeting considered that further research in this field should be cost-effective. The following specific recommendations for further research were made.

\subsection{RECOMMENDATIONS}

2.5.1 Methods for prediction of trends should be further developed and validated, and be used in a more systematic manner in the monitoring and evaluation of leprosy control. In each instance the least complex method should be used which is valid for that particular purpose.

2.5.2 Simulation models for leprosy control should be further developed by incorporating current simulation techniques and decision sciences methodology. The development and testing of these models should be a multi-disciplinary effort, involving at least scientists responsible for epidemiological field research and control of leprosy, and scientists with experience in epidemiological modelling.

2.5.3 In implementing the above recommendations, optimal use should be made of existing epidemiological data sets for leprosy.

\section{Assessment of the leprosy problem}

\subsection{INTRODUCTION}

The issues relating to the assessment of the leprosy problem were limited to the quantitative estimation of prevalence and incidence of leprosy.

\subsection{DEFINITION OF A CASE}

The Sixth Report of the WHO Expert Committee on Leprosy (Technical Report Series $768,1988)$ defined a case of leprosy as 'a person showing clinical signs of leprosy with or without bacteriological confirmation of the diagnosis, and requiring chemotherapy'. There was a consensus that for quantitative assessment a leprosy case was a person needing chemotherapy. It is recognized that it is difficult to predict which lesions would self-heal and that keeping such persons under observation would pose problems for assessment; theref ore they should be considered as cases needing chemotherapy. Relapses should also be considered as cases but those who had completed chemotherapy should no longer be considered as cases. 


\subsection{ASSESSMENT OF INCIDENCE}

As the incidence cannot be assessed by direct measurement it should be inferred by indirect methods. Detection rates should be taken as the best estimate of incidence until better methods are developed.

\subsection{ASSESSMENT OF PREVALENCE}

The prevalence is an index which is more amenable to direct measurement. There is a need for methods of estimating prevalence which would be rapid and economical. The period following the introduction of MDT poses special problems because of the rapid decline in prevalence, improved ascertainment of cases, and increased sensitivity of diagnosis.

3.4.1 Total population examination is both extremely expensive and unnecessary.

3.4.2 Sample surveys are the standard method of obtaining information about disease prevalence-however, the required sample sizes are extremely large because of the uneven distribution of leprosy. Sample surveys in leprosy are expensive and both sampling and non-sampling errors are likely to be large. Therefore such sample surveys should be carried out with extreme care and in exceptional circumstances.

3.4.3 Methods of estimating prevalence through extrapolation from registered cases should especially be considered. The number of cases in registers have been shown to correlate with estimated cases, as revealed by sample surveys. The extrapolation factor will be different following the introduction of MDT.

3.4.4 Rapid community surveys is another economical means to assess prevalence. In this procedure an intensive eff ort is made to educate the population of the community on the signs of leprosy and the efficacy of modern drugs. The survey team then visits the community, and with the help of key local personnel, individuals with suspected signs are examined in a central place.

\subsection{ASSESSMENT IN THE LATER PHASES OF ELIMINATION}

Towards the elimination stage, priorities will shift towards the rehabilitative needs of patients, both under treatment and those who have completed treatment but have disability. The emphasis will be on the assessment of disability due to leprosy.

\section{Basic data requirements for the evaluation of leprosy control programmes}

\subsection{INTRODUCTION}

The Report of the WHO Study Group on Epidemiology of Leprosy in Relation to Control (TRS 716, 1985) listed standardized indicators to evaluate leprosy control activities. Many of these were found to be extremely useful during the initial few years of MDT implementation. However, the number of indicators is far too many for routine applications under field conditions in most leprosy endemic countries. By now the efficacy of MDT regimens for both $\mathrm{PB}$ and $\mathrm{MB}$ has been clearly established by leprosy control programmes. There is now need to focus on the most essential indicators which could assist in decision making for leprosy programmes implementing MDT.

Essential indicators need to be relevant to assess the magnitude of the leprosy problem 
and the effectiveness of leprosy control measures. Based on recommendations made during the Consultation on Technical and Operational Aspects of Leprosy held at Male, Maldives, in June 1990, six indicators are recommended as the most essential. A number of other important indicators which are considered useful are suggested for application in programmes which are capable of collecting and reporting the data required for the compilation of these indicators. These are listed as optional indicators.

\subsubsection{Essential indicators}

The essential indicators have been identified based on their relevance and feasibility at both national and peripheral levels. Data required to calculate these indicators should be collected by all leprosy control programmes. It is recommended that these indicators are calculated once a year. The first two indicators should not only be reported as rates, but also in absolute numbers.

4.1.1.1 Prevalence rate. The prevalence rate is defined as the number of registered cases at the end of the year divided by the population in which the cases have occurred. The population should refer to the most recent, consistent and reliable source. This indicator reflects the magnitude of the problem, and helps in planning and evaluation of control measures. It is also necessary for evaluating workload, but in that respect it must be interpreted in conjunction with the new case detection.

4.1.1.2 Case detection rate. This rate is defined as the number of new cases detected during a year divided by the population in which the cases have occurred. This indicator is the most appropriate to estimate the true incidence of the disease in a given population, when analysed in conjunction with the proportion of disabled patients (grade 2) among newly detected cases. This should be specified by the type of the disease (PB/MB).

4.1.1.3 Disability rate among newly-detected cases. This is defined as the proportion of newly-detected cases with grade 2 disability among the total number of newly-detected cases during the year. This indicator reflects the effectiveness of the programme in terms of early case finding and the level of community awareness of the disease.

4.1.1.4 MDT coverage. This is defined as the proportion of cases registered for multidrug therapy at any time during the year, among the total number of cases appearing on the register during the year. The denominator is the total number of cases that was on the treatment (=monotherapy + MDT) register at any time during the year.

This indicator reflects the programme performance in achieving full MDT coverage and helps to set targets.

4.1.1.5 Number of patients having completing MDT during the years. In those programmes in which the recording and reporting system does not yet allow the collection of such information or the duration of MDT is ill-defined, the number of patients completing MDT during the year could be used as a proxy indicator.

Where possible, the MDT completion rate should be calculated. It is defined as the proportion of patients who have completed their MDT among patients expected to complete their MDT. This must be calculated separately for MB and PB cases.

This indicator is important to evaluate the patient compliance to treatment as well as the programme performance in providing adequate MDT.

4.1.1.6 Number of patients relapsing after MDT. Careful recording and reporting of relapses is important for use as a crude marker of treatment failures which could indicate the need for a special study. 


\subsubsection{Optional indicators}

These indicators may be used for specific purposes wherever possible. Additional resources may be needed for collection of some data required for these indicators.

4.1.2.1 Prevalence rate by type- $P B / M B$

4.1.2.2 Proportion of children among newly-detected cases

4.1.2.3 New cases, specified by mode of detection

4.1.2.4 Incidence of new disability among registered cases

4.1.2.5 MDT coverage in new patients

\subsubsection{Indicators for use in the later phases of elimination}

In this situation the most relevant indicator is the total number of new cases and relapsed cases detected during the year. In this era indicators reflecting the prevalence of leprosy related disabilities will be important because of the shift in priorities towards monitoring and managing rehabilitative needs of patients. 


\section{Appendix}

This table illustrates how the indicators are calculated. It is not intended to replace existing reporting forms.

\begin{tabular}{|c|c|c|c|c|c|c|c|}
\hline & \multicolumn{3}{|c|}{ Population } & \multicolumn{4}{|c|}{ Year } \\
\hline & \multirow{2}{*}{$\begin{array}{c}\text { Registered } \\
\text { cases at } \\
\text { the end of } \\
\text { the previous } \\
\text { year }\end{array}$} & \multicolumn{2}{|c|}{$\begin{array}{l}\text { New cases detected } \\
\text { during the year }\end{array}$} & \multirow[b]{2}{*}{$\begin{array}{c}\text { Cases on } \\
\text { MDT } \\
\text { during } \\
\text { the year }\end{array}$} & \multirow[b]{2}{*}{$\begin{array}{l}\text { Completed } \\
\text { MDT } \\
\text { during } \\
\text { the year }\end{array}$} & \multirow[b]{2}{*}{$\begin{array}{l}\text { Registered } \\
\text { cases at } \\
\text { the end } \\
\text { of the year }\end{array}$} & \multirow[b]{2}{*}{$\begin{array}{c}\text { Relapses } \\
\text { after } \\
\text { completion } \\
\text { of MDT }\end{array}$} \\
\hline & & $\begin{array}{c}\text { With } \\
\text { disability } \\
\text { grade } 2\end{array}$ & $\begin{array}{c}\text { Without } \\
\text { disability } \\
\text { grade } 2\end{array}$ & & & & \\
\hline PB & a & $\mathrm{b}$ & c & $\mathrm{e}$ & $\mathrm{j}$ & $\mathrm{p}$ & 1 \\
\hline MB & $\mathrm{f}$ & $\mathrm{g}$ & $\mathrm{h}$ & $\mathrm{i}$ & $\mathrm{k}$ & q & $\mathrm{m}$ \\
\hline Total & $a+f$ & $b+g$ & $\mathrm{c}+\mathrm{h}$ & $\mathrm{e}+\mathrm{i}$ & $\mathrm{j}+\mathrm{k}$ & $\mathrm{p}+\mathrm{q}$ & $1+m$ \\
\hline
\end{tabular}

1. Prevalence (rate)

- prevalence $\mathrm{p}+\mathrm{q}=\frac{\mathrm{p}+\mathrm{q}}{\text { population }} \times 10,000$

2. Case detection (rate)

- total number of new cases $=b+g+c+h$

- percentage of $\mathrm{MB}$ cases $=\frac{\mathrm{g}+\mathrm{h}}{\mathrm{b}+\mathrm{g}+\mathrm{c}+\mathrm{h}} \times 100$

- case detection rate $\quad=\frac{\mathrm{b}+\mathrm{g}+\mathrm{c}+\mathrm{h}}{\text { population }} \times 10,000$

3. Disability rate among newly detected cases

$=\frac{b+g}{b+g+c+h} \times 100$

4. MDT coverage

$=\frac{\mathrm{e}+\mathrm{i}}{\mathrm{a}+\mathrm{f}+\mathrm{b}+\mathrm{g}+\mathrm{c}+\mathrm{h}} \times 100$

5. Number of relapses after MDT during the year (Absolute number)

$=1+\mathrm{m}$ 